\title{
On-Line Calibration Method for Stereovision Systems Used in Vehicle Applications
}

\author{
Sergiu Nedevschi, Cristian Vancea, Tiberiu Marita, Thorsten Graf
}

\begin{abstract}
This paper presents an on-line calibration method of the absolute extrinsic parameters of a stereovision system suited for vision based vehicle applications. The method uses as prior knowledge the intrinsic parameters and the relative extrinsic parameters (relative position and orientation) of the two cameras, which are calibrated using off-line procedures. These parameters are remaining unchanged if the two cameras are mounted on a rigid frame (stereo-rig). The absolute extrinsic parameters are defining the position and orientation of the stereo system relative to a world coordinate system. They must be calibrated every time after mounting the stereorig in the vehicle and are subject to changes due to static (variable load) and dynamic (acceleration, bumpy road) factors. The proposed method is able to perform on-line the estimation of the absolute extrinsic parameters by driving the car on a flat and straight road, parallel with the longitudinal lane markers. The edge points of the longitudinal lane markers are extracted after a $2 \mathrm{D}$ image classification process and reconstructed by stereovision in the stereo-rig coordinate system. After filtering out the noisy 3D points the normal vectors of the world coordinate system axes are estimated in the stereo-rig coordinate system by $3 \mathrm{D}$ data fitting. The output of the method is the height and the orientation of the stereo rig relative to the world coordinate system.
\end{abstract}

\section{INTRODUCTION}

$\mathrm{V}$ ISION based sensors are gaining greater importance over the active sensors (radars, laser scanners, etc.) in the field of driving assistance systems due to their decreasing costs and increasing hardware processing capabilities. Moreover, tasks such as the lane detection are one of the exclusive privileges of computer vision.

The measuring capabilities of vision sensors with monocular cameras are reduced because corresponding methods are relying on some prior knowledge of the scene (such as flat road assumption, constant pitch angle, etc.), which cannot be always fulfilled in real scenarios. However, a full 3D reconstruction of the driving environment is possible when using a stereovision system.

Manuscript received: 20-th March 2005. This work was supported by Volkswagen A.G., Germany.

Sergiu Nedevschi, Cristian Vancea, Tiberiu Marita, are with Technical University of Cluj-Napoca, Computer Science Department, 400020 ClujNapoca, ROMANIA (phone: +40-264-401457; fax: +40-264-594491; email: Sergiu.Nedevschi\{, Cristian.Vancea, Tiberiu.Marita\}@cs.utcluj.ro.

T. Graf, is with Volkswagen AG, Electronic Research, 38436 Wolfsburg, GERMANY (+49-5361-9-29420, fax: +49-5361- 9-72837; email: thorsten.graf@volkswagen.de.
In the second case, the quality of the reconstruction is strictly dependent on the estimation (calibration) accuracy of the following parameters:

- Internal parameters of the stereo-system: intrinsic parameters (focal length and the principal point positions) and relative position and orientation of the two cameras (relative extrinsic parameters).

- Absolute extrinsic parameters of the stereo-system: position and orientation of the stereo-rig (usually the left camera coordinate system) relative to a world coordinate system in which the measurements are reported.

The quality of the internal parameters influences the accuracy of the essential and fundamental matrix and consequently the position of the epipolar lines [1], which is essential in the stereo correlation process. Their wrong estimation can lead to a lack of correlated points or to false correlations which is disastrous to the whole stereo reconstruction process. The quality of the absolute extrinsic parameters is linked only to the accuracy of the $3 \mathrm{D}$ measurements relative to the world coordinate system.

Regarding the intrinsic parameters calibration there are many general purpose methods found in the literature [2][5], which can be applied off-line. Most of them are estimating the parameters by minimizing the projection errors of a set of control points from a calibration object/pattern, with known structure, against the detected $2 \mathrm{D}$ images of the same control points. Multiple views of the calibration object are taken and the accuracy of the results usually increases with the total number of considered control points. The Bouguet method implemented in the Caltech Camera Calibration Toolbox [5] has also the possibility to estimate the relative extrinsic parameters of a stereovision system. Moreover, many integrated stereovision systems [6], [7] are delivered with off-line calibration tools able to estimate the internal parameters of the stereo-system.

Regarding the absolute extrinsic parameters estimation, the existing methods can be grouped in two categories: offline methods and on-line methods.

For the off-line methods the principle is the same: minimizing the projection error of some 3D control points with known positions in the world coordinate system (measurement coordinate system). Therefore all the absolute extrinsic parameters can be estimated (rotation matrix and translation vector [1]). Some general purpose methods [5], [8] are using the same calibration object as for the intrinsic parameters estimation. Such an approach is suited only for near range applications (e.g. indoor visual navigation of 
robots). Dedicated methods for vision based driving assistance systems are using painted markers in known positions on a flat road surface, as in [9] and [10]. Because the markers are placed in a large depth range, the obtained accuracy for far range detection is higher compared to the general purpose methods.

Regarding the on-line methods, a dedicated calibration object with known geometry is no more available. Nevertheless, some prior knowledge about the calibration scene structure must be known in advance. The most facile approach is the usage of a planar road surface with some painted markers. The absence of a calibration object limits the number of detected parameters. In [11] and [12] Weber has proposed two ground plane-fitting methods based on the residual disparity of road points, in order to estimate a subset of parameters of a stereo camera mounted on a vehicle: the height, the pitch angle and a sort of roll angle, but now yaw angle. In [9] a method for on-line adjustment of the extrinsic parameters based on painted markers on the car's hood was introduced, but the extrinsic parameters are relative to the ego-car coordinate system and not to a global, road related one.

In this paper we present a fast on-line calibration method able to estimate the orientation and height of the stereocameras, related to the ego-vehicle world coordinate system. The world coordinate system has the $\mathbf{O X}$ and $\mathbf{O Z}$ axes in the road plane with $\mathbf{O Z}$ coinciding with the longitudinal axis of the ego-vehicle (Fig. 3). Its origin is the projection of the ego-vehicle's front wheel axis central point on the road plane and is moving with the car along the road. The height of the stereo-system and its orientation relative to this world coordinate system are influenced by variable load of the car. Therefore performing an on-line calibration of the absolute extrinsic parameters for the initial conditions of a specific vehicle-setup is essential in order to perform a complete reconstruction of the driving environment (implying 3D stereo-reconstruction, road obstacle separation, obstacle detection, as in [13] and [15]). Furthermore, dynamic variations of the ego vehicle's orientation and position relative to a lane related world coordinate system can be performed by specialized lane detection modules as in [14], [15].

\section{PROBLEM STATEMENT}

Considering the intrinsic parameters and the relative extrinsic parameters of both cameras already known (because they can be estimated off-line using dedicated calibration methods), it is possible to perform 3D reconstruction [13] in the left camera coordinate system also referred as Camera Reference Frame (CRF). The purpose of a vision based vehicle application is to perform the reconstruction in a world coordinate system (referred as World Reference Frame - WRF). In order to do that, the rotation matrix and translation vector of the left camera $\left(\mathbf{R}_{C}^{\text {left }}, \mathbf{T}_{C}^{\text {left }}\right)$, with respect to WRF, have to be determined. Furthermore, using the relative extrinsic parameters $\left(\mathbf{T}_{r e l}\right.$, $\left.\mathbf{R}_{r e l}\right)$, right camera position and orientation can be calculated, thus obtaining the complete calibration of the stereovision system's absolute extrinsic parameters:

$\left\{\begin{array}{l}\mathbf{T}_{C}{ }^{r i g h t}=\mathbf{T}_{C}{ }^{\text {left }}+\mathbf{R}_{C}{ }^{\text {left }} \cdot \mathbf{T}_{r e l} \\ \mathbf{R}_{C}{ }^{r i g h t}=\mathbf{R}_{C}{ }^{\text {left }} \cdot \mathbf{R}_{r e l}\end{array}\right.$

When the rig is mounted into the car it has a dedicated mounting position which will be the same, with very small errors, even if the rig is mounted or un-mounted repeatedly. We also consider that the cameras are tightly fixed on the rig, and their relative position and orientation will not change if not specifically wanted. The position of the left camera (CRF) in WRF is defined as the translation vector $\mathbf{T}_{C}^{\text {left }}=\left[\begin{array}{lll}T_{C X} & T_{C Y} & T_{C Z}\end{array}\right]^{\mathrm{T}}$. The lateral offset $T_{C X}$ has a relative meaning to the car longitudinal axis; the longitudinal offset $T_{C Z}$ has a relative meaning to the car front wheel axis. Both parameters can be measured (with a few $\mathrm{cm}$ errors) or estimated (using the off-line calibration procedure), so there is no need to estimate them on-line. Therefore, the only remaining problem to be solved is the estimation of the rotation matrix $\left(\mathbf{R}_{C}^{\text {left }}\right)$ of the CRF and also its height $\left(T_{C Y}\right)$, which is highly dependent on the car load.

\section{OVERVIEW OF THE METHOD}

The proposed solution is based on $3 \mathrm{D}$ coordinates of reconstructed points (in CRF) from lane markings' edges painted on the road surface. Therefore this method does not require calibration objects with a known structure, nor calibration patterns, but a suitable environment respecting the following constraints:

- flat road surface with no curvature or inclination;

- presence of clear longitudinal lane markings on each side of the car and no other objects on the road;

- longitudinal axis of the car should be parallel with the road longitudinal axis and also with the lane markings all the time (no change in car's moving direction is allowed);

- for better results it is recommended to have continuous lane markings, slow speed and (as much as possible) a canonical placement of the cameras on the rig and small values for yaw and roll angles of the left camera, related to road plane.

The on-line calibration process is performed along a fixed number of consecutive pairs of images, captured while driving the car. For each pair, two major steps are applied:

- first step consists in extracting the current lane markings' edge points. The only important edges are those parallel with the longitudinal axis of the road, thus each lane marking has only two useful edges;

- second step takes as input the 3D coordinates of the previously classified edge points, reconstructed in CRF using a fast and high accuracy stereoreconstruction algorithm [13]. The output will be the rotation matrix $\mathbf{R}_{C}^{\text {left }}$ and the height $T_{C Y}$ of the CRF related to WRF. 


\section{CLASSIFICATION OF THE ROAD MARKING POINTS}

Road features are searched only through a 2D analysis on the left image. Possible lane marking edges are searched as pairs of segments respecting the following constraints:

- there is a limited distance among them;

- similar intensity difference is encountered between the inner-bright and outer-dark sides;

- are situated bellow the vanishing point;

- their extensions intersect the vanishing point.

The vanishing point position is determined using an initial vanishing point estimation method, followed by a vanishing point position tracking throughout the pairs of captured images. The initial estimation is also used whenever the vanishing point tracking fails, as means to reinitialize the tracking procedure.

\section{A. Initial vanishing point estimation}

The lines containing segments filtered with respect to the first two constraints are plotted on two interlaced grids formed by square cells of size equal to the permitted error in estimating the vanishing point position (Fig. 1). Each cell has an associated value, which is incremented with the length of any segment (without extension part) that passes through it. Finally the cells that have the maximum values in the two grids, as well as the cells that have values equal or between the two maximums, are selected and their center of weight is returned as an approximation of the vanishing point position. The two maximums must have neighboring positions otherwise the vanishing point is not validated.

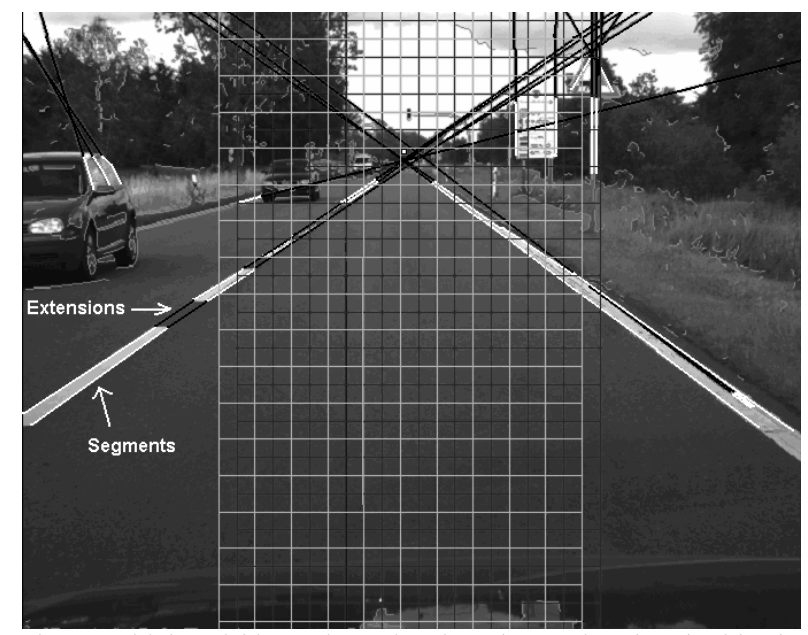

Fig. 1. Initial vanishing point estimation: the two interlaced grids; the filtered segments before applying vanishing point discrimination (white) and their extensions (black)

\section{B. Vanishing point tracking}

Once we know the vanishing point position, it can be used as an extra constraint for the returned lane marking segments, as their extensions must be passing close to it.

In the end of the whole feature selection algorithm, the segments classified as lane markings' edges are known and their equations in the image plane are used to determine the vanishing point position for the next frame, as their intersection:

$\left\{\begin{array}{c}x_{v p}=\text { slope }_{1} \cdot y_{v p}+\text { offset }_{1} \\ \cdots \\ x_{v p}=\text { slope }_{n} \cdot y_{v p}+\text { offset }_{n}\end{array}\right.$

where $\left(x_{v p}, y_{v p}\right)$ is the unknown vanishing point position and slope $_{n}$, offset $_{n}$ are parameters describing $n^{\text {th }}$ segment equation.

Applying Least Squares we obtain $\left(x_{v p}, y_{v p}\right)$ as the point having the minimum sum of the squared distances to every line containing lane markings' segments.

\section{Selection of the proper lane markings}

Normally all lane markings can be used in the calibration process, but for accuracy reasons we selected only those belonging to the current lane. Considering that the stereo system is forward oriented, current lane markings will appear by the two sides of the vertical line that passes through the vanishing point. They are selected by their orientations, considering the vertical line as reference and the positive angles, counter clockwise:

- the right current lane markings are found having the minimum positive angles;

- the left current lane markings are found having the maximum negative angles.

The angles found for the current lane are tracked from frame to frame, using a polar histogram (Fig. 2) containing the results from a certain number of previous frames. In case the markings from one side of the current lane are lost, nothing is returned for that frame.

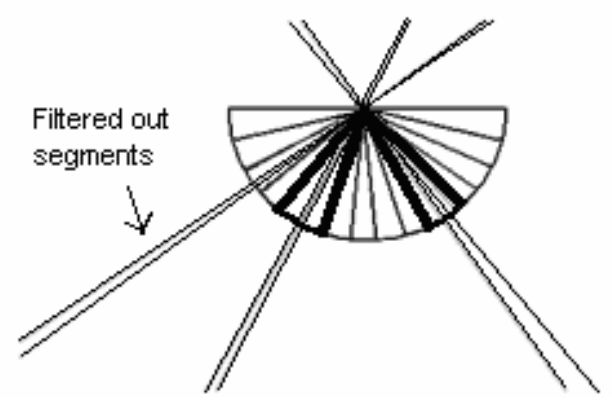

Fig. 2. Polar histogram used to track current lane markings' segments. Segments not passing the thick black-sectors are filtered out.

\section{ESTIMATION OF THE ABSOLUTE EXTRINSIC PARAMETERS}

All points of each segment classified as belonging to the current lane makings are further reconstructed in CRF and their $3 \mathrm{D}$ coordinates are used in the process of estimating the rotation matrix $\left(\mathbf{R}_{C}^{\text {left }}\right)$ and height $\left(T_{C Y}\right)$.

We will further consider that the axes of WRF are $\mathbf{O X}_{W}$, $\mathbf{O Y}{ }_{W}, \mathbf{O Z}_{W}$ and the axes of $\mathrm{CRF}$ are $\mathbf{O} \mathbf{X}_{C}, \mathbf{O Y} \mathbf{Y}_{C}, \mathbf{O Z}_{C}($ Fig. 3). 


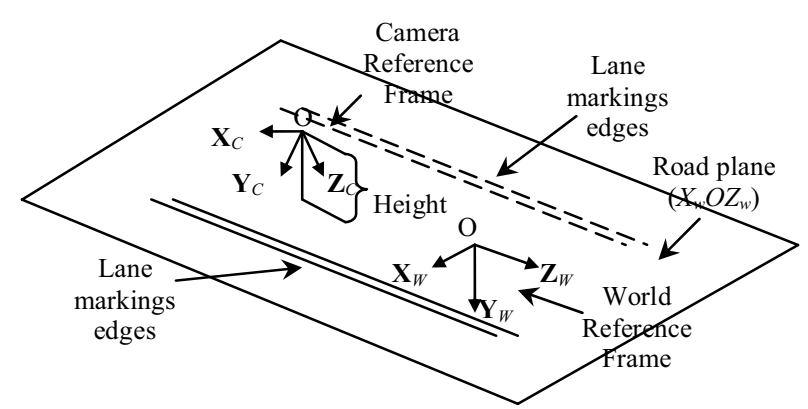

Fig. 3. WRF $\left(\mathrm{O} X_{W} Y_{W} Z_{W}\right)$ and $\mathrm{CRF}\left(\mathrm{O} X_{C} Y_{C} Z_{C}\right)$ - scene overview.

Given a point position in CRF, described by vector $\mathbf{P}_{C}$, its position in WRF will be given by vector $\mathbf{P}_{W}$ according to the following formula:

$\mathbf{P}_{W}=\mathbf{R}_{C}^{l e f t} \cdot \mathbf{P}_{C}+\mathbf{T}_{C}^{l e f t}$

$T_{C X}$ and $T_{C Z}$ components of $\mathbf{T}_{C}^{\text {left }}$ vector are considered to be known (they can be measured or estimated), so it only remains to estimate $\mathbf{R}_{C}^{\text {left }}$ and $T_{C Y}$, where:

$$
\mathbf{R}_{C}^{l e f t}=\left[\begin{array}{lll}
\mathbf{O} \mathbf{X}_{W}^{\mathbf{T}} & \mathbf{O Y} & \mathbf{O Z} \\
W
\end{array}\right]^{\mathbf{T}}
$$

and $\mathbf{O} \mathbf{X}_{W}, \mathbf{O} \mathbf{Y}_{W}, \mathbf{O Z}_{W}$ are the normalized vectors corresponding to the WRF axes in the camera reference frame (Fig. 4). In order to have a better view, the normalized vectors of the WRF were translated in Fig. 4 in the center of CRF. This process does not affect the rotation matrix.

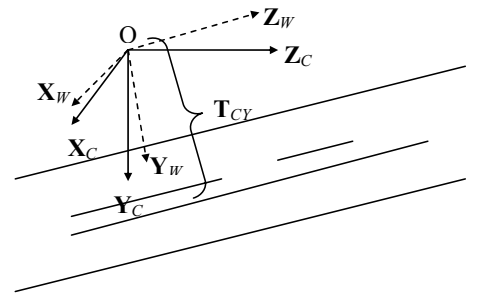

Fig. 4. WRF $\left(\mathrm{O} X_{W} Y_{W} Z_{W}\right)$ orientation in $\mathrm{CRF}\left(\mathrm{O} X_{C} Y_{C} Z_{C}\right)$.

The problem of computing $\mathbf{R}_{C}^{\text {left }}$ (4) is reduced to estimating only $\mathbf{O} \mathbf{Y}_{W}$ and $\mathbf{O} \mathbf{Z}_{W}$, because $\mathbf{O} \mathbf{X}_{W}$ is the cross product of the first two:

$\mathbf{O X} \mathbf{X}_{W}=\mathbf{O Y}_{W} \times \mathbf{O Z}_{W}$

As the car is driven parallel with the lane markings, their $3 \mathrm{D}$ reconstructed edges in CRF should be parallel with $\mathbf{O Z}_{W}$ axis, so its orientation can be obtained simply by estimating the normalized direction of any of the edges. In order to obtain a robust result, we calculated the average orientation of all reconstructed edges.

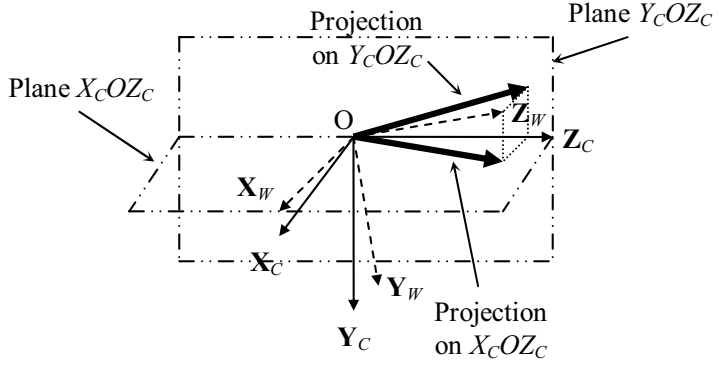

Fig. 5. Projection of lane marking segments on planes $X_{C} O Z_{C}$ and $Y_{C} O Z_{C}$. Each reconstructed segment should have identical orientation as $\mathbf{O Z}$ axis.

The 3D orientation of an edge can be calculated from its projections on planes $X_{C} O Z_{C}$ and $Y_{C} O Z_{C}$ (Fig. 5). Actually each edge is represented by a set of $n$ reconstructed points in CRF. Their projections on $X_{C} O Z_{C}$, respectively $Y_{C} O Z_{C}$ are constrained by the following set of equations:

$\left\{\begin{array}{c}a_{X O Z} \cdot Z_{1}+b_{X O Z}=X_{1} \\ \ldots \\ a_{X O Z} \cdot Z_{n}+b_{X O Z}=X_{n}\end{array} \quad\left\{\begin{array}{c}a_{Y O Z} \cdot Z_{1}+b_{Y O Z}=Y_{1} \\ \cdots \\ a_{Y O Z} \cdot Z_{n}+b_{Y O Z}=Y_{n}\end{array}\right.\right.$

where $\mathbf{P}_{C}{ }^{k}=\left[\begin{array}{lll}X_{k} & Y_{k} & Z_{k}\end{array}\right]^{\mathrm{T}}$. Initially, we filter out points having depth and lateral offsets outside of a given range (outside our Space-Of-Interest (SOI) [15]). Afterwards we interpolate the remaining points through a weighted leastsquares process applied on (6), where $a_{X O Z}, b_{X O Z}, a_{Y O Z}, b_{Y O Z}$ are the unknowns. Finally the 3D orientation of the edge is obtained as the following vector:

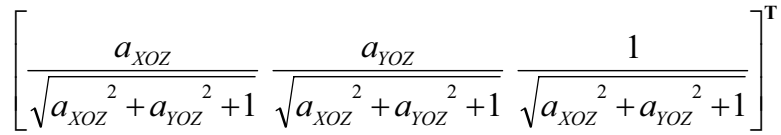

The weight associated to each point is inverse proportional with its reconstruction error and respects the following formula:

Weight $_{k}=\operatorname{Max} W-\frac{\operatorname{Max} W-\operatorname{Min} W}{\operatorname{Max} E} \cdot$ Error $_{k}$

where weights are in range $\operatorname{Min} W$ to $\operatorname{Max} W, \operatorname{Max} E$ is the maximum reconstruction error allowed (points with greater errors are removed) and Error $k_{k}$ is the estimated reconstruction error for current point. Considering the case of a canonical stereo-system, it is possible to derive a formula which estimates the reconstruction error in CRF by simply knowing the correlation error $(\Delta c)$, the focal distance $(f)$ and the baseline $(b)$ :

$\operatorname{Error}_{k}=\left|\frac{Z_{k} \cdot \Delta c \cdot \sqrt{X_{k}^{2}+Y_{k}^{2}+Z_{k}^{2}}}{f \cdot b+\Delta c \cdot Z_{k}^{2}}\right|$ 
We were able to use this approximation also in a general camera configuration with small relative angles, because the differences between estimated errors, for a canonical configuration compared to our general configuration, were up to $150 \mathrm{~mm}$ in the case of points situated at $10 \mathrm{~m}$ height, $100 \mathrm{~m}$ ahead and $8 \mathrm{~m}$ lateral offset (extreme limit of our SOI). These are maximum errors and only a very small percentage of the points will encounter such coordinates/errors.

The weighted least-squares process is repeated several times, each time the points being filtered out if their fitting error is greater than a fixed percentage of the average fitting error. We imposed several conditions for stopping the iterations:

- number of iterations equals a certain value concerning the speed;

- number of remaining points becomes lower than a certain percentage of their initial number;

- average fitting error drops under a certain value (based on experimental results);

- average fitting error does not converge.

Once the orientation of an edge is obtained using (7), it is compared with the average $\mathbf{O Z}_{W}$ axis obtained from a fixed number of previously captured image frames. A filtering threshold is defined in terms of a fixed distance and a fixed maximum error allowed at that distance:

Threshold $=\tan ^{-1} \frac{\text { MaxAllowedError }}{\text { FixedDist }}$

This approach is based on the fact that lane markings' edges are parallel with $\mathbf{O Z}_{W}$ axis and their orientation remains constant from frame to frame, assuming the car does not change its heading direction.

The remaining edge points are known to be points from road plane and respect the following constraints:

$$
\left\{\begin{array}{l}
A \cdot X_{1}+B \cdot Y_{1}+C \cdot Z_{1}=H \\
\quad \cdots \\
A \cdot X_{n}+B \cdot Y_{n}+C \cdot Z_{n}=H \\
A^{2}+B^{2}+C^{2}=1
\end{array}\right.
$$

where $H$ is the height of the plane and $\mathbf{n}=\left[\begin{array}{lll}A & B & C\end{array}\right]^{\mathrm{T}}$ is the normal to the plane. The above system of equations can be solved using a weighted least-squares process, in which the weights are calculated according to (8). The result is a plane-fitting of all road points, thus obtaining the normal vector $\mathbf{n}=\mathbf{O} \mathbf{Y}_{W}$ and the height $H=T_{C Y}$. This process is performed several times, until one of the above stated stopping conditions is fulfilled.

Once $\mathbf{O} \mathbf{Y}_{W}$ and $\mathbf{O} \mathbf{Z}_{W}$ axes are estimated, we calculate $\mathbf{O X}_{W}$ using (5). Therefore we obtain the rotation matrix $\mathbf{R}_{C}^{\text {left }}$ according to (4). Finally, we apply a cleaning process on rotation matrix elements, based on Singular Value Decomposition (SVD), in order to eliminate possible axis distortions appeared due to precision errors in computations or imperfect 3D reconstruction of points in CRF. This final stage insures an orthogonal shape for the rotation matrix [5].

As the on-line calibration is performed on several consecutive frames, the results are averaged over frames, in order to avoid unstable results due to small car oscillations or small changes in heading direction.

\section{EXPERIMENTS}

We performed several experiments while driving the car on an approximately flat road with clear lane markings. The intrinsic parameters of the cameras and relative extrinsic parameters were considered known, as they were obtained using an off-line calibration process. Table I contains several initialization parameters we used while performing the on-line calibration of the absolute extrinsic parameters.

TABLE I

CONSTANTS USED FOR THE EXPERIMENTAL SETUP

\begin{tabular}{|c|c|c|}
\hline Symbol & Value & Description \\
\hline$[\operatorname{Min} W, \operatorname{Max} W]$ & 0.2 pixels & $\begin{array}{l}\text { correlation error in (9); it was } \\
\text { chosen as the most frequent } \\
\text { correlation error obtained after } \\
\text { performing several experiments } \\
\text { allowed weight range in (8) }\end{array}$ \\
\hline $\operatorname{Max} E$ & $0.5 \mathrm{~m}$ & maximum reconstruction error in (8) \\
\hline $\begin{array}{l}\text { MaxAllowedError } \\
\quad \text { FixedDist }\end{array}$ & $\begin{array}{l}550 \mathrm{~mm} \\
50 \mathrm{~m}\end{array}$ & $\begin{array}{l}\text { maximum allowed error in }(10) \\
\text { distance for maximum allowed error } \\
\text { in }(10)\end{array}$ \\
\hline
\end{tabular}

The tests were performed on 50 consecutive frames. The estimated rotation matrix and height were compared with those obtained using an off-line calibration process. Results concerning height and derived rotation angles (pitch, yaw and roll) corresponding to the computed rotation matrix, are presented in Fig. 6.

As it may be noticed, all four parameters were close to the off-line estimated values with offsets induced by different vehicle load, compared to the off-line setup, proving method stability and accuracy. Tests performed on other sequences revealed the same small error's range. The yaw angle estimation is highly dependant on driver's ability to drive the car parallel with the lane markings.

Regarding the implementation of extrinsic parameters estimation phase, its complexity respects the following formula:

$$
\text { NoComputations }=2 \cdot \sum_{i=1}^{n} I_{i} \cdot P_{i}+2 \cdot I \cdot P
$$

where $n$ is the number of lane classified marking edges, $I_{i}$ is the number of weighted least-squares iterations performed when estimating $e d g e_{i}$ 's direction, $P_{i}$ is number of points from $e^{d g e_{i}, I}$ is the number of weighted least-squares iterations performed for road plane fitting and $P$ is the number of points involved in road plane fitting.

The average processing time needed for extracting the lane markings' edges was about $3.9 \mathrm{~ms}$ (644x512 image) while for the extrinsic parameters estimation about $0.2 \mathrm{~ms}$, resulting a total time of $4.1 \mathrm{~ms}$. Tests were deployed on a 2.6 GHz Intel Pentium IV Processor . 


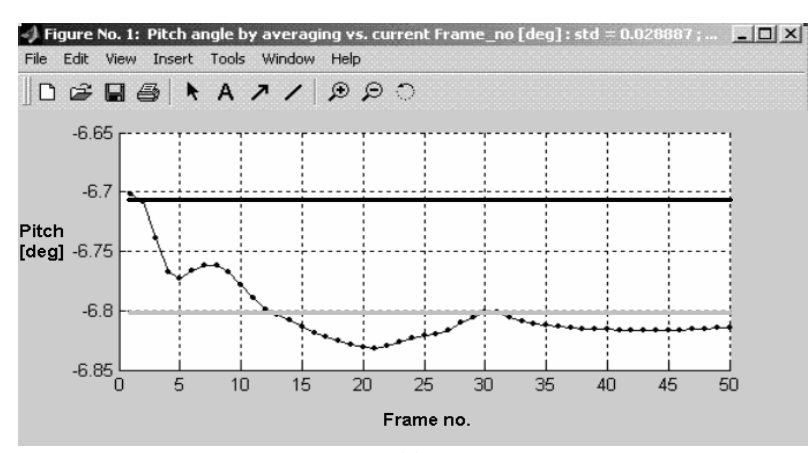

(a)

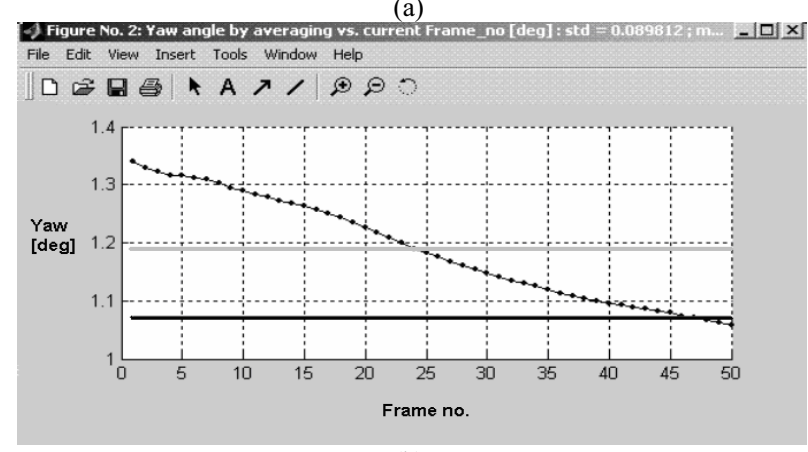

(b)

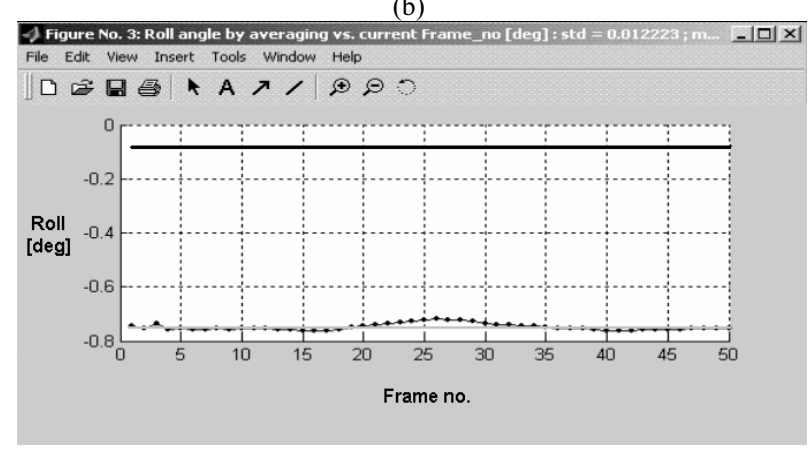

(c)

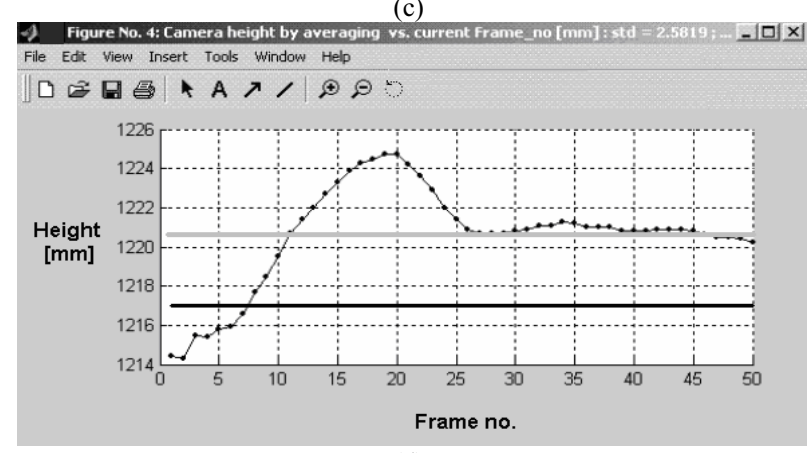

(d)

Fig. 6. Experimental results on 50 consecutive frames: a) Pitch angle by averaging rotation matrix on previous frames; b) Yaw angle by averaging rotation matrix on previous frames; c) Roll angle by averaging rotation matrix on previous frames; d) Height by averaging results on previous frames. (dots - estimated values; thick gray-line mean value; thick black-line - values of the off-line calibration).

\section{CONCLUSIONS}

In this paper was proposed an on-line calibration method of the absolute extrinsic parameters characterizing a stereovision system, suited for vehicle applications. The intrinsic and relative extrinsic parameters of the cameras are considered to be known as they can be estimated using a high precision off-line calibration procedure.

The process does not require any calibration objects with a known structure, nor calibration patterns, but only a suitable environment consisting in a flat and straight road with clearly painted lane markings. Such an environment may be found on highways or marked roads, so it does not require the construction of special calibration fields. The results were very close to those obtained using the off-line calibration, with offsets determined by static load factors for the used car-setup.

\section{REFERENCES}

[1] E. Trucco, "Introductory techniques for 3D Computer Vision", Prentice Hall, 1998.

[2] Tsai, R. Y.(1987), "A versatile camera calibration technique for highaccuracy $3 \mathrm{D}$ machine vision metrology using off-the-shelf TV cameras and lenses". IEEE Journal of Robotics and Automation, RA3(4)/1987: pp.323-344.

[3] Zhang Z (1999), "Flexible Camera Calibration By Viewing a Plane From Unknown Orientations." International Conference on Computer Vision (ICCV'99), Corfu, Greece, September 1999, pp. 666-673.

[4] Heikkila, J.; Silven, O. (1997), "A four-step camera calibration procedure with implicit image correction," Proc. IEEE Computer Society Conf., 1997 pp.1106-1112.

[5] J.Y. Bouguet, Camera Calibration Toolbox for Matlab, $\mathrm{http}: / /$ www.vision.caltech.edu/bouguetj/calib doc/

[6] Videre Design (Home page), http://www.videredesign.com

[7] Tyzx (Home page), http://www.tyzx.com/

[8] N. Kämpchen, , U. Franke, R. Ott, "Stereo vision based pose estimation of parking lots using 3D vehicle models", IEEE Intelligent Vehicle Symposium, 2002, pp. 459- 464.

[9] S. Ernst, C. Stiller, J. Goldbeck, C. Roessig, "Camera Calibration for Lane and Obstacle detection", IEEE Inteligent Transportation Systems, 1999, pp. 356-361.

[10]A. Broggi, M. Bertozzi, A. Fascioli "Self-Calibration of a Stereo Vision System for Automotive Applications", IEEE Conf. on Robotics and Automation, Seoul, Korea, May 2001, vol.4, pp. 3698-3703.

[11]J. Weber, M. Atkin, "Further results on the use of binocular vision for highway driving", Transportation Sensors and Controls: Collision Avoidance, Traffic Management and ITS, Proc. SPIE 2592, 1995.

[12] J.Weber, D. Koller, Q.-T. Luong and J. Malik, "An integrated stereobased approach to automatic vehicle guidance", Fifth International Conference on Computer Vision, Boston 1995, Collision Avoidance and Automated Traffic Management Sensors, Proc. SPIE 2592, 1995.

[13]S. Nedevschi, R. Schmidt, T. Graf, R. Danescu, D. Frentiu, T. Marita, F. Oniga, C. Pocol, "High Accuracy Stereo Vision System for Far Distance Obstacle Detection", in Proc. of IEEE Intelligent Vehicles Symposium, Parma, Italy, June 14-17, 2004, pp.161-166.

[14] S. Nedevschi, R..Schmidt, T. Graf, R. Danescu, D. Frentiu, T. Marita, F. Oniga, C. Pocol, "3D Lane Detection System Based on Stereovision", in Proc. of IEEE Intelligent Transportation Systems Conference (ITSC), Washington, USA, October 4-6, 2004, pp. 292297.

[15]S. Nedevschi, R. Danescu, T. Marita, F. Oniga, C. Pocol, S. Sobol, T. Graf, R. Schmidt, "Driving Environment Perception Using Stereovision", Procedeeings of IEEE Intelligent Vehicles Symposium, (IV2005), June 2005, Las Vegas, USA, pp.331-336. 